\title{
A Carbon Nanofilament-Bead Necklace
}

\author{
Li Song, ${ }^{*} \dagger$ Alexander W. Holleitner, ${ }^{\ddagger}$ Huihong Qian, ${ }^{\S}$ Achim Hartschuh, ${ }^{\S}$ Markus Döblinger,$\S$ \\ Eva M. Weig, ${ }^{\dagger}$ and Jörg P. Kotthaus ${ }^{\dagger}$ \\ Center for NanoScience (CeNS) und Fakultät für Physik, Ludwig-Maximilians-Universität, \\ Geschwister-Scholl-Platz 1, 80539 Munich, Germany, Walter Schottky Institut (WSI), Technische Universität \\ München, Am Coulombwall 3, 85748 Munich, Germany, and Department Chemie und Biochemie, \\ Ludwig-Maximilians-Universität, Butenandtstrasse 11, 81377 Munich, Germany
}

Received: March 3, 2008; Revised Manuscript Received: April 14, 2008

\begin{abstract}
Carbon nanofilaments with carbon beads grown on their surfaces were successfully synthesized reproducibly by a floating catalyst CVD method. The nanofilaments hosting the pearl-like structures typically show an average diameter of about $60 \mathrm{~nm}$, which mostly consists of low-ordered graphite layers. The beads with diameter range 150-450 $\mathrm{nm}$ are composed of hundreds of crumpled and random graphite layers. The mechanism for the formation of these beaded nanofilaments is ascribed to two nucleation processes of the pyrolytic carbon deposition, arising from a temperature gradient between different parts of the reaction chamber. Furthermore, the Raman scattering properties of the beaded nanofilaments have been measured, as well as their confocal Raman G-line images. The Raman spectra reveal that that the trunks of the nanofilaments have better graphitic properties than the beads, which is consistent with the HRTEM analysis. The beaded nanofilaments are expected to have high potential applications in composites, which should exhibit both particleand fiber-reinforcing functions for the host matrixes.
\end{abstract}

\section{Introduction}

During the past decade, carbon nanomaterials have been attracting great interest in the research community because of their remarkable electronic and mechanical properties. ${ }^{1-7} \mathrm{Nu}-$ merous carbon nanostructures have been synthesized, including carbon filaments and fibers,,${ }^{1,2}$ fullerenes, ${ }^{3}$ carbon nanotubes, ${ }^{4}$ onions, ${ }^{5}$ and nanocones. ${ }^{6,7}$ Due to their low density and high elastic modulus, carbon filaments have large potential commercial applications in reinforcement composites for the space and aircraft industries as well as high-performance sports equipment. ${ }^{8}$ To this end, carbon filaments principally act to increase the strength of composites by deflecting or bridging cracks, which are induced by the applied stress. ${ }^{9,10}$ It is wellknown that the interfacial strengths are very important for such filament-reinforcing composites, and they should be as high as possible in order to transfer the maximum load between the host matrixes and the filaments. In order to improve the above interfacial strength, several attempts have been made to develop the degree of oxidation of the carbon filament surface ${ }^{11,12}$ or to grow filaments with special morphologies. ${ }^{13-15}$ It is expected that filaments with beaded structure should be the ideal reinforcing agent because the nearby beads could provide a grasping point for releasing the slippage between the host matrixes and the filaments. Recently, carbon nanotubes coated with carbon-glass beads were observed in arc discharge products, ${ }^{16}$ and short carbon beads with protruding cones were produced by a catalyst method. ${ }^{17}$ However, it is still a challenge to synthesize beaded filaments by a low-temperature process.

\footnotetext{
* To whom correspondence should be addressed. E-mail: li.song@ physik.uni-muenchen.de.

$\dagger$ Center for NanoScience (CeNS) and Fakultät für Physik, LudwigMaximilians-Universität.

*WSI, Technische Universität München.

$\S$ Department Chemie and Biochemie, Ludwig-Maximilians-Universität.
}

In this paper, carbon nanofilaments with carbon beads grown on their surfaces were successfully synthesized reproducibly by thermally decomposing methane $\left(\mathrm{CH}_{4}\right)$ at $1100{ }^{\circ} \mathrm{C}$ in a floating catalyst chemical vapor deposition (CVD) system. The nanofilaments hosting the pearl-like structures typically show an average diameter about $60 \mathrm{~nm}$. These trunks mostly consist of lowordered graphite layers. The beads with the diameter range of 150-450 nm are composed of hundreds of crumpled and randomly oriented graphite layers. We have employed confocal Raman imaging, along with scanning electron microscopic and transmission electron microscopic analysis, to reveal the necklacelike structure of the nanofilament. The subsequent Raman spectra taken from its trunk and bead regions demonstrate a short-range ordering of graphite layers in the nanofilament. The mechanism for the formation of these beaded nanofilaments is ascribed to two nucleation processes of pyrolytic carbon deposition, arising from a temperature gradient between different parts of the CVD reaction chamber.

\section{Experimental Section}

Our experimental setup is a two-stage furnace system, and a quartz tube is fitted inside the furnace to act as the reaction chamber. The catalyst powder (ferrocene, $\mathrm{Fe}\left(\mathrm{C}_{5} \mathrm{H}_{5}\right)_{2}$ ) was sublimated first at a temperature of $60-70{ }^{\circ} \mathrm{C}$ and carried by a flowing argon (50-150 sccm) and methane (5-8 sccm) mixture into the second furnace through the quartz tube. The reaction temperature of the second furnace was $1100{ }^{\circ} \mathrm{C}$. Then, the methane was decomposed to synthesize carbon products under the assistance of the catalyst. The as-grown products were carried out from the furnace and deposited onto silicon wafers or other sample carriers, which were placed at the end of the quartz tube. The typical deposition time of the beaded nanofilaments was about 10-15 min for enhancing the yield of asgrown samples. 


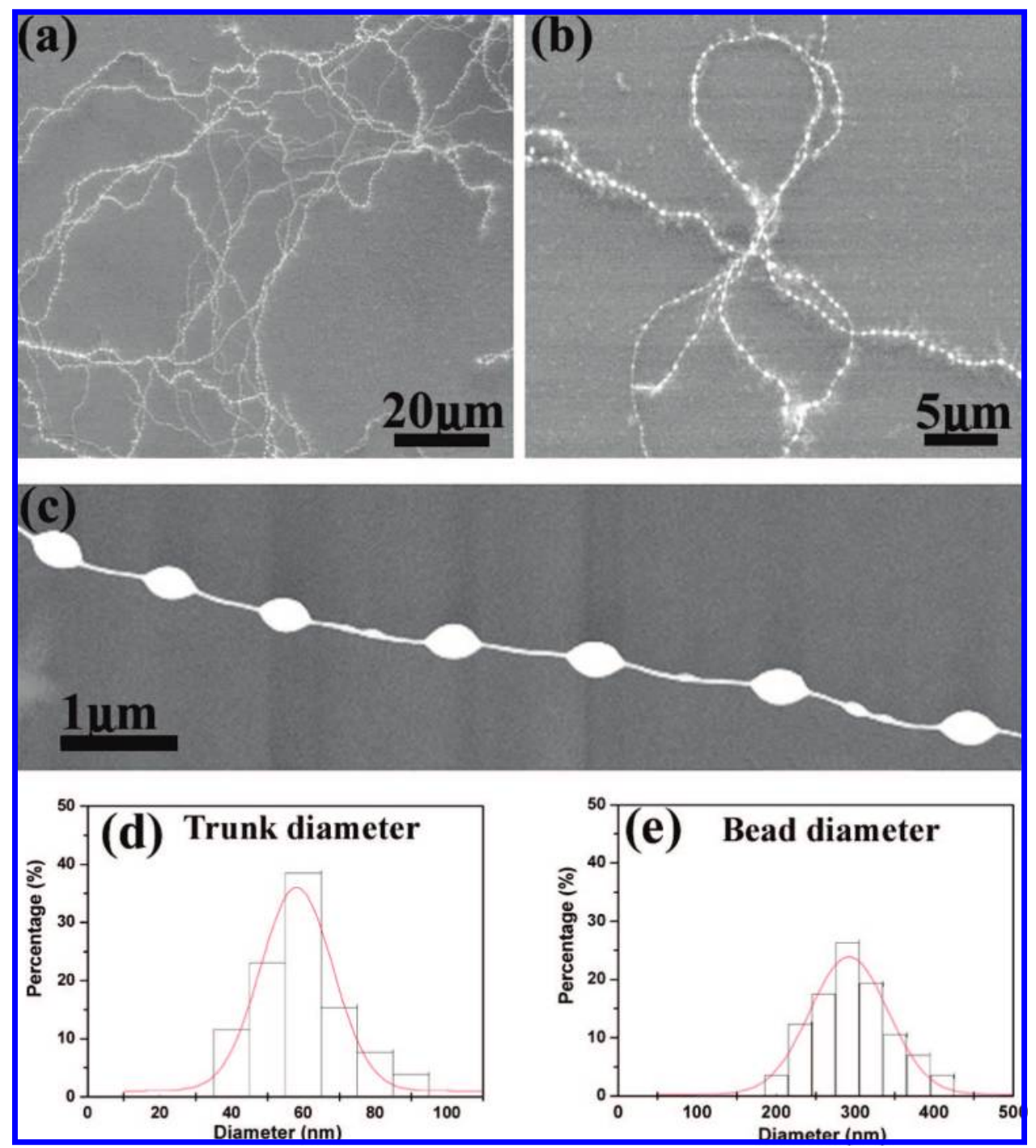

Figure 1. ( $a, b)$ Low and high magnification SEM images of beaded nanofilaments. (c) SEM image clearly showing a single nanofilament with pearl-like structure. (d, e) Histograms showing the diameter distribution for the trunks and beads of the nanofilament, as well as Gaussian fits.

Scanning electron microscopy (SEM; LEO DSM-982) and transmission electron microscopy (TEM; FEI Titan 80-300) were applied to characterize the as-grown products. The optical properties of beaded nanofilaments were investigated by utilizing a confocal Raman setup. The corresponding setup is based on an inverted optical microscope with $x$ and $y$ scanning stages for scanning a transparent sample. The Raman signal is detected by single-counting avalanche photodiodes after passing bandpass filters centered at $700 \mathrm{~nm}$.

\section{Results and Discussion}

Structure and Morphology. A typical morphology of the carbon nanofilaments as grown by our floating CVD method is depicted in the SEM images shown in Figure 1. The floating CVD method provides a high yield of the nanofilaments (Figure 1a,b), which exhibit smooth surfaces as shown in Figure 1c. Estimated from several tens of SEM images of our products, we find that the trunks of these nanofilaments typically have an average diameter of $60 \mathrm{~nm}$ with a narrow Gaussian distribution (Figure 1d), while the beads grown on the filament's surface show a diameter range from 150 to $450 \mathrm{~nm}$ (Figure 1e). The distance between two beads varies from several hundreds of nanometers to several micrometers. For further investigation, the products were deposited on a copper grid for TEM characterization.

Figure $2 \mathrm{a}$ is a typical TEM image of a single nanofilament, while Figure $2 b-d$ gives enlarged views of several parts of the filament. It appears that the filament has a bead-necklace geometry with some particles on its surface. Energy dispersive X-ray spectroscopy (EDX; Figure 2e) reveals that the nanofilaments are composed of carbon, decorated with some iron particles that are attributed to catalyst residue. More details are shown in the following high resolution TEM views recorded from different parts of the beaded nanofilament (see Figure 3).

Figure 3 a clearly shows that there is a continuous core inside the nanofilament, and a bead is formed on its surface (see arrows). In addition, iron particles with very small diameter appear on the surface of the trunk as well as within the beads (black dots in Figure 3a,d). It is apparent from the high resolution TEM views in Figure $3 b$ that the center column is mostly composed of partially ordered graphite layers, while crumpled and randomly oriented graphite layers appear on the body of the beads and the whole nanofilament surface. From evaluation of diffraction patterns and several HRTEM images, we found a distance of about $0.36 \mathrm{~nm}$ which corresponds to the plane distance of graphite layers. Figure $3 \mathrm{c}$ also reveals that 


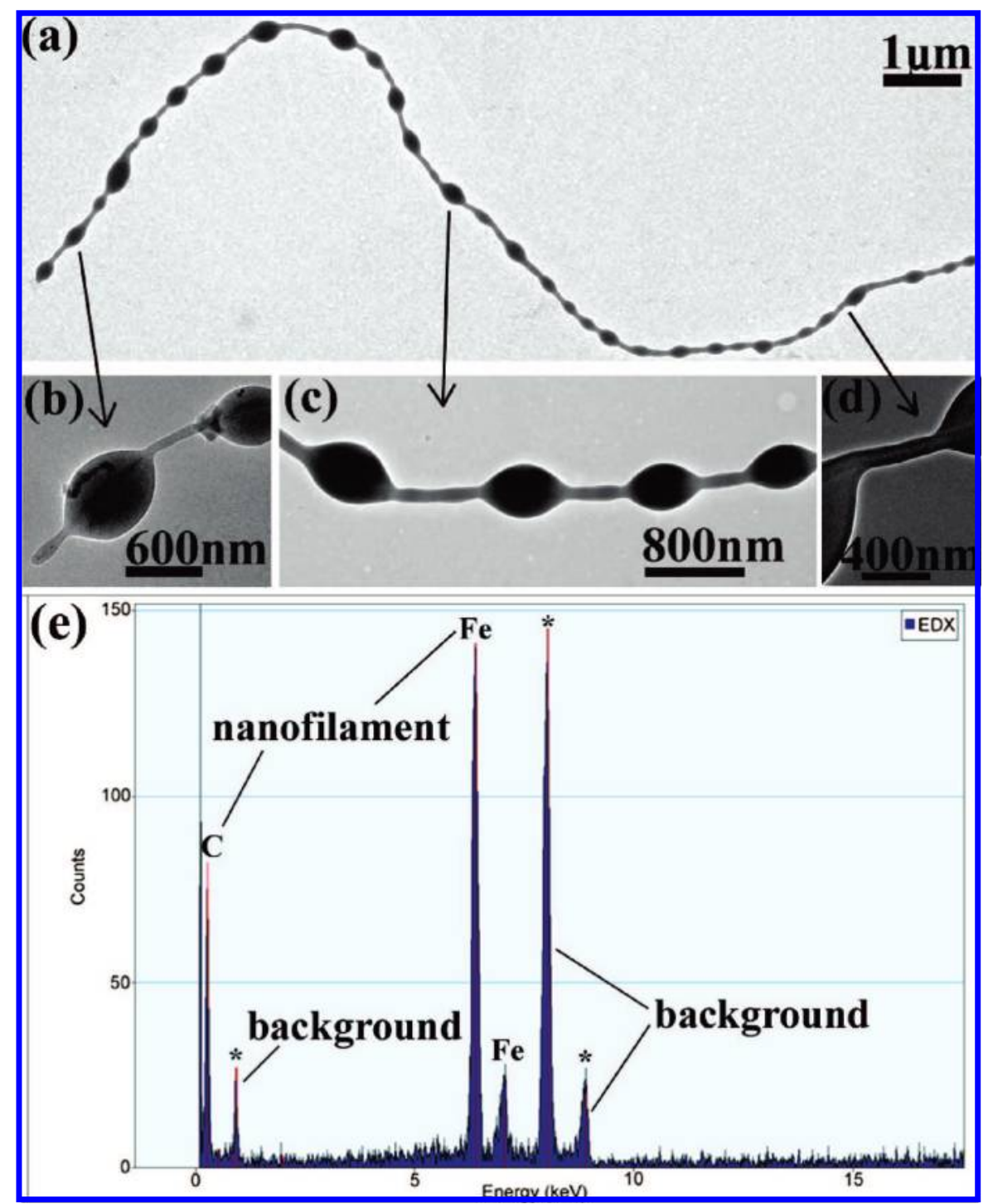

Figure 2. $(a-d)$ TEM images of a single beaded nanofilament with pearl-like structure. (e) The corresponding EDX spectrum of the nanofilaments, in which the resonances indicated by asterisks are from the copper grid.

iron particles are encapsulated into several graphite layers. The (110) crystal planes of $\alpha$-iron with a typical plane distance of $0.20 \mathrm{~nm}$ are detected here. This finding indicates a good crystallization of the iron particles. ${ }^{18}$ A selected-area electron diffraction (SAED) pattern is shown in Figure 3e. It reveals diffuse reflections corresponding to a partially ordered stacking of graphite layers along the filament direction. The diffuse rings can be attributed to in-plane distances of disordered graphite. On the other hand, some clear reflections can be indexed according to $\alpha$-iron. ${ }^{19}$

Confocal Raman Spectroscopy. In order to verify the shortrange ordering structures of graphite in the beaded nanofilaments, we utilize confocal Raman spectroscopy. ${ }^{20-22}$ Figure 4 shows such confocal Raman images and corresponding Raman spectra of the as-grown beaded nanofilaments, measured with a laser excitation wavelength of $632.8 \mathrm{~nm}(1.96 \mathrm{eV})$. In Figure $4 \mathrm{a}-\mathrm{c}$, Raman images are detected by utilizing a band-pass filter centered at $700 \mathrm{~nm}$ corresponding to Raman shifts between 1420 and $1620 \mathrm{~cm}^{-1}$. As is known for graphite structures, Raman signals at about $1600 \mathrm{~cm}^{-1}$ result from $\mathrm{C}=\mathrm{C}$ stretching modes forming the so-called G-band. The G-line images in Figure 4a,b clearly show the pearl-like structure of the nanofilaments, which exactly resolves the bead and trunk positions (bright points and garnet connection). The Raman spectra are subsequently recorded with a higher spatial resolution on the bead and trunk positions (1, 2 and 3, 4 in Figure 4d), as displayed in Figure 4c. We find that the Raman spectra of the trunks consist of three major groups of peaks. The first group includes the peaks at about 1340 and $1609 \mathrm{~cm}^{-1}$, which correspond to the D-mode and G-mode of graphite, respectively. ${ }^{23,24}$ The second group of peaks (at $2615-3195 \mathrm{~cm}^{-1}$ ) is related to the $\mathrm{D}^{*}$-mode and $\mathrm{G}^{*}$ mode of graphite. The last group of peaks around $5768 \mathrm{~cm}^{-1}$ is due to the higher-order mode of graphite. ${ }^{25,26}$ As compared with the Raman spectrum recorded from the trunks, the D- and G-peaks appear weaker for the beads, while their $\mathrm{D}^{*}$ - and $\mathrm{G}^{*}$ lines show extremely broad contributions. It is noteworthy that the higher-frequency peak from the higher-order mode of graphite $2 \mathrm{D}+2 \mathrm{G}$ only appears in the Raman spectra of the trunks. This finding suggests that the trunks of the beaded nanofilaments have better graphitic properties than the beads, which is consistent with the above HRTEM observation in Figure 3. Since the size of the beads is in the range of the diameter of the focal spot of our Raman microscope, i.e., about $300 \mathrm{~nm}$, they are expected to block the silicon Raman peak at $520 \mathrm{~cm}^{-1}$ which only appears in the Raman spectra of the trunks.

Growth Mechanism. There are several possibilities of how carbon filaments form at different morphologies. ${ }^{16,17,27-29}$ Based on the above TEM, SEM, and Raman observations, we attribute 


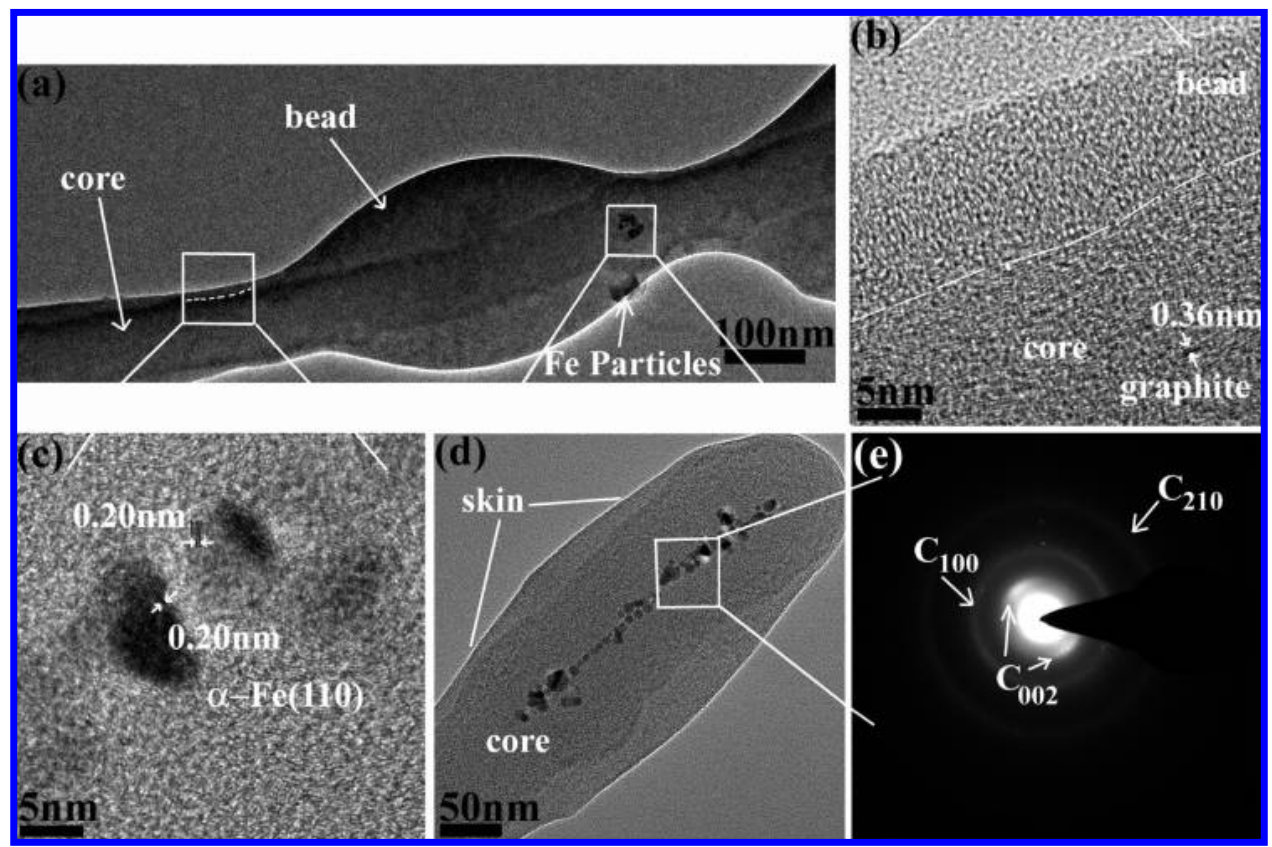

Figure 3. $(\mathrm{a}-\mathrm{d})$ High resolution TEM views of the beaded nanofilament. Lines and arrows are guides to the eyes. (e) The selected-area electron diffraction (SAED) recorded from one part of the nanofilament in (d).
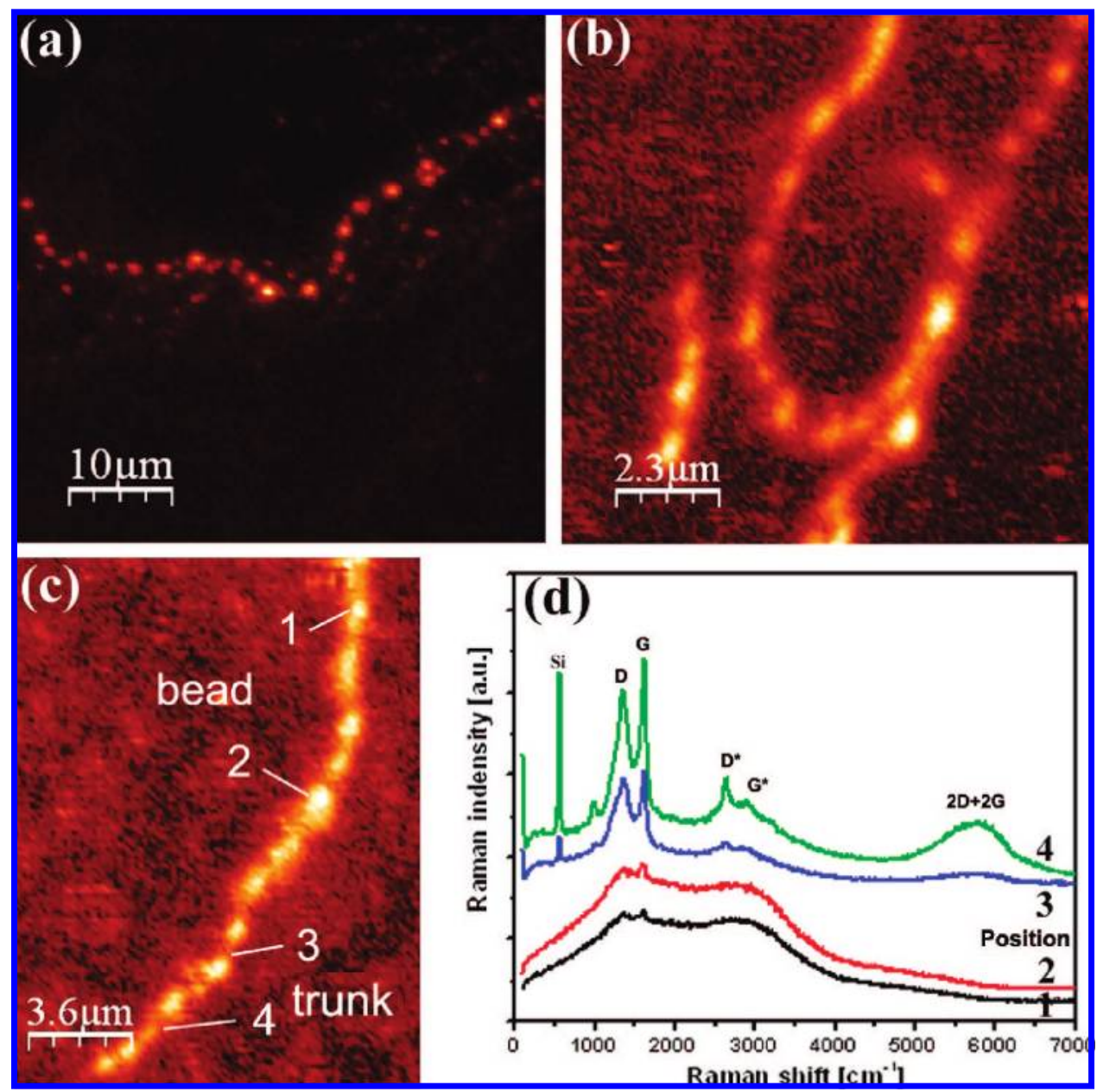

Figure 4. $(\mathrm{a}-\mathrm{c})$ Confocal Raman G-line images of the pearl-like nanofilaments representing the intensity between 1420 and $1620 \mathrm{~cm}^{-1}$. $(\mathrm{d})$ Corresponding Raman spectra taken from the bead positions (1 and 2), and the trunk positions (3 and 4) in (c).

the formation of the beaded nanofilaments with pearl-like structure to two nucleation processes during the carbon deposition process. We assume that both processes are driven by a temperature gradient between different parts of our CVD oven
(Figure 5e). A simple schematic model of the growth process for a single beaded necklace is shown in Figure 5. At the beginning of the growth process (Figure 5a), methane is thermally decomposed, and filaments with small diameter are 


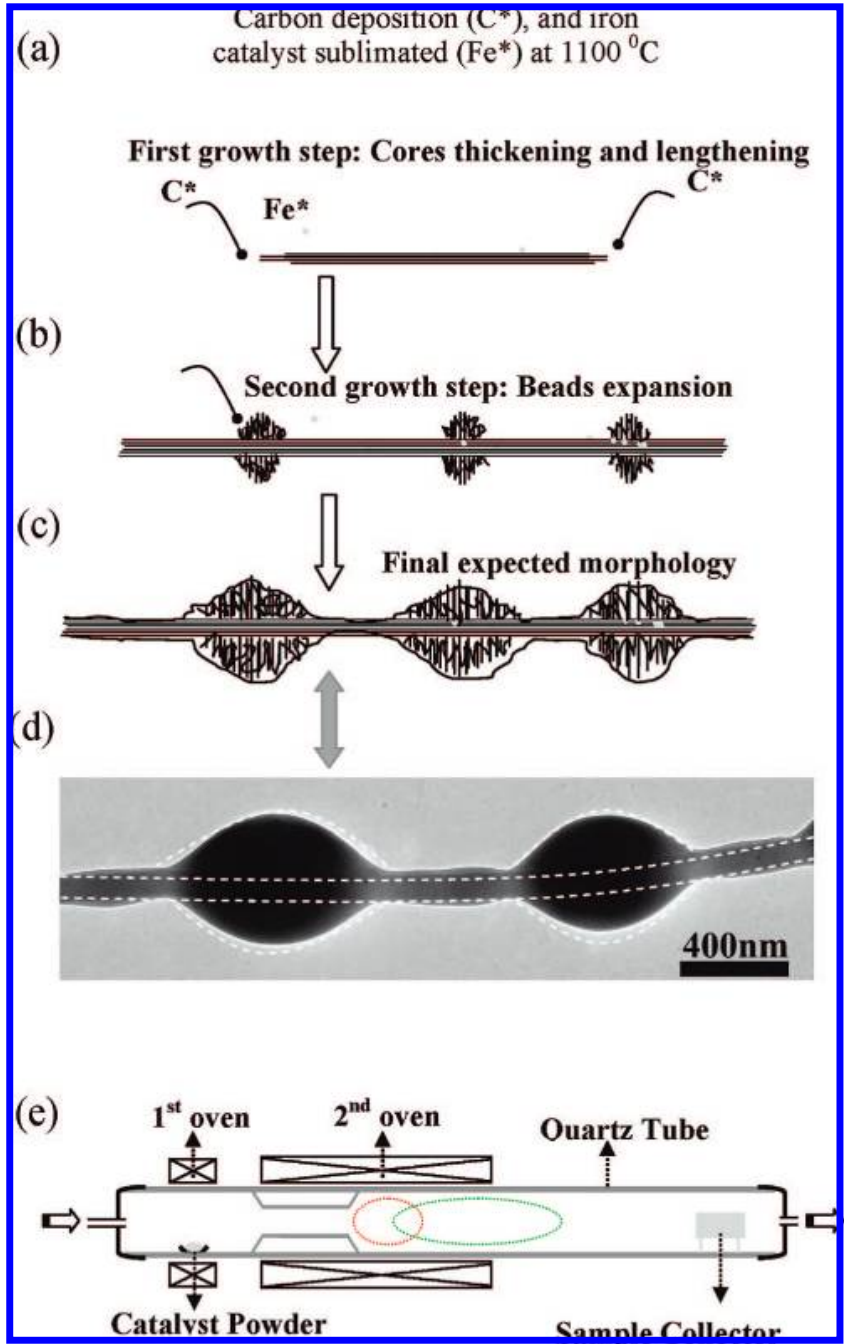

Figure 5. $(a-c)$ Schematic growth mode of beaded nanofilaments with pearl-like structure. (d) The corresponding TEM images of one beaded nanofilament with similar shape in our experiments. (e) Schematic diagram of the experimental two-stage furnace setup.

quickly synthesized in the high-temperature region $\left(1100^{\circ} \mathrm{C}\right)$. This is the first nucleation process, and it is similar to the ones previously reported. ${ }^{14-16,30-32}$ In the second step, the as-grown primary filaments are carried away from the highest temperature region with the gas flow. During the corresponding transport time, the temperature of the samples decreases, and the surface free energy of the filaments is changed as the temperature decreases. ${ }^{33-36}$ The latter results in the second nucleation process of the pyrolytic carbon deposition. The pyrolytic carbon deposition periodically nucleates to form biconical deposit morphologies, as shown in Figure 5b. As the pyrolytic carbon deposition progresses with time, the biconical structure dimensions keep increasing to create bead morphologies with a smooth surface. Finally, at a region of much lower temperature, the pyrolytic carbon deposition becomes more random, which results in the thin disordered graphite skin on the whole surface of the beaded nanofilament that is visible in Figure 3a,d. In general, the observed bead structure is consistent with the expected structure from the above model indicated by dashed lines in Figure 5d. It is likely that contaminant species (for example, oxygen) inside the oven may also have an effect on the forming process of the beads as the temperature is lowered. ${ }^{37-39}$ Oxygen could etch the as-grown nanofilaments to create beadlike geometry during our CVD deposition process. The effect of Fe crystals in the formation of the beads is not clear, since the crystals can be found both on the surface and inside the nanofilaments.

\section{Conclusions}

In summary, we have demonstrated a new process for a simple and reproducible growth of carbon nanofilaments with pearl-like structure by thermally decomposing $\mathrm{CH}_{4}$ in a floating iron catalyst system. The nanofilaments typically have diameters of $60 \mathrm{~nm}$, while the beads show a diameter range of 150-450 nm, which is mostly composed of many crumpled graphite layers. The Raman scattering properties of the beaded nanofilaments have been measured, as well as their confocal Raman G-line images. The Raman spectra reveal that the trunks of the nanofilaments have better graphitic properties than the beads, a finding which is also supported by the HRTEM analysis. The synthesis method of beaded filament will provide promising materials for designing high-mechanical reinforced composites.

Acknowledgment. We are grateful for financial support from CeNS, the Nanosystems Initiative Munich (NIM), and the DFG through Grant SFB486 TPA1. L.S. acknowledges the support of the Alexander von Humboldt Foundation and discussions with Prof. Sishen Xie and Dr. Lijie Ci.

\section{References and Notes}

(1) Gogotsi, Y.; Libera, J. A.; Kalashnikov, N.; Yoshimura, M. Science 2000, 290, 317.

(2) Bacon, R. J. Appl. Phys. 1960, 31, 283.

(3) Kroto, H. W.; Heath, J. R.; O'Brien, S. C.; Curl, R. F.; Smalley, R. E. Nature 1985, 318, 163.

(4) Iijima, S. Nature 1991, 354, 56.

(5) Ugarte, D. Nature 1992, 359, 707.

(6) Sattler, K. Carbon 1995, 33, 915.

(7) Xia, W.; Schlüter, O. F. K.; Muhler, M. Carbon 2004, 42, 2751.

(8) Dresselhaus, M. S.; Dresslhaus, G.; Sugihara, K.; Spain, I. L.; Goldberg, H. A. Graphite fibers and filament; Springer-Verlag: Berlin, 1989.

(9) Figueiredo, J. I.; Bernardo, C. A.; Baker, R. T. K.; Hüttinger, K. J. Carbon fiber, Filaments and composites; Kluwer Academic Publishing: Dordrecht, 1990.

(10) Rand, B. In Watt, W.; Perov, B. V. Handbook of composites, Vol. 1, Strong Fibers; Elsevier: Amsterdam, 1985; p 495.

(11) Donnet, J.-B.; Bansal, R. C. Carbon Fibers; Marcel Dekker Inc.: New York, 1984.

(12) Hüttinger, K. J. Adv. Mater. 1990, 2, 349

(13) Fu, L.; Liu, Z. M.; Liu, Y. Q.; Han, B. X.; Hu, P. G.; Cao, L. C.; Zhu, D. B. Adv. Mater. 2005, 17, 217.

(14) Hao, Y. J.; Wagner, J. B.; Su, D. S.; Jin, G. Q.; Guo, X. Y. Nanotechnology 2006, 17, 2870.

(15) Singjai, P.; Wongjamras, A.; Yu, L. D.; Tunkasiri, T. Chem. Phys. Lett. 2002, 366, 51.

(16) de Heer, W. A.; Poncharal, P.; Berger, C.; Gezo, J.; Song, Z. M.; Bettini, J.; Ugarte, D. Science 2005, 307, 907.

(17) Jacobsen, R. L.; Monthioux, M. Nature 1997, 385, 211.

(18) Jin-Phillipp, N. Y.; Rühle, M. Phys. Rev. B 2004, 70, 245421.

(19) Wie, D. C.; Cao, L. C.; Fu, L.; Li, X. L.; Wang, Y.; Yu, G.; Liu, Y. Q. Adv. Mater. 2007, 19, 386.

(20) Hartschuh, A.; Qian, H. H.; Meixner, A. J.; Anderson, N.; Novotny, L. Nano Lett. 2005, 5, 2310.

(21) Ferrari, A. C.; Robertson, J. Raman spectroscopy in carbons: from nanotubes to diamond; The Royal Society: London, 2004.

(22) Harris, P. J. F. Carbon 2007, 45, 229.

(23) Ferrari, A. C.; Robertson, J. Philos. Trans. R. Soc. London, A 2004, $362,2477$.

(24) Tan, P. H.; Hu, C. Y.; Dong, J.; Shen, W. C.; Zhang, B. F. Phys. Rev. B 2001, 64, 214301.

(25) Reich, S.; Thomsen, C. Philos. Trans. R. Soc. London, A 2004, $362,2271$.

(26) Tan, P. H.; Dimovski, S.; Gogotsi, Y. Philos. Trans. R. Soc. London,. A 2004, 362, 2289.

(27) Murayama, H.; Maeda, M. Nature 1990, 345, 791.

(28) Kato, T.; Kusakabe, K.; Morooka, S. J. Mater. Sci. Lett. 1992, 11, 
(29) Ci, L. J.; Wei, J. Q.; Wei, B. Q.; Xu, C. L.; Liang, J.; Wu, D. H. J. Mater. Sci. Lett. 2000, 19, 21.

(30) Monthioux, M.; Allouche, H.; Jacobsen, R. L. Carbon 2006, 44, 3183.

(31) Harris, P. J. F. Carbon 2007, 45, 229.

(32) Delhaès, P. Carbon 2002, 40, 641.

(33) Chen, C. J.; Back, M. H. Carbon 1979, 17, 175.

(34) Je, J. H.; Lee, J. Y. Carbon 1984, 22, 317.

(35) Shi, R.; Li, H. J.; Yang, Z.; Kang, M. K. Carbon 1997, 35, 1789.
(36) Hu, Z. J.; Reznik, B.; Zhang, W. G.; Gerthsen, D.; Hüttinger, K. J. Carbon 2003, 41, 749 .

(37) Hu, Z. J.; Hüttinger, K. J. Carbon 2002, 40, 624.

(38) Allouche, H.; Monthioux, M.; Jacobsen, R. L. Carbon 2003, 41, 2897.

(39) Allouche, H.; Monthioux, M. Carbon 2005, 43, 1265.

JP8018588 